Research Article

\title{
Further Results about Seeking for the Exact Solutions of the Nonlinear $(2+1)$-Dimensional Jaulent-Miodek Equation
}

\author{
Jingsen $\mathrm{Hu}$ and Jianming Qi \\ School of Business, Shanghai Dianji University, Shanghai 201306, China \\ Correspondence should be addressed to Jianming Qi; qijianmingdaxia@163.com
}

Received 21 May 2021; Revised 25 June 2021; Accepted 10 July 2021; Published 29 July 2021

Academic Editor: Wen-Xiu Ma

Copyright (C) 2021 Jingsen Hu and Jianming Qi. This is an open access article distributed under the Creative Commons Attribution License, which permits unrestricted use, distribution, and reproduction in any medium, provided the original work is properly cited.

\begin{abstract}
Nonlinear science is a great revolution of modern natural science. As a result of its rise, the various branches of subjects characterized by nonlinearity have been developed vigorously. In particular, more attention to acquiring the exact solutions of a wide variety of nonlinear equations has been paid by people. In this paper, three methods for solving the exact solutions of the nonlinear $(2+1)$-dimensional Jaulent-Miodek equation are introduced in detail. First of all, the exact solutions of this nonlinear equation are obtained by using the $\exp (-\phi(z))$-expansion method, tanh method, and sine-cosine method. Secondly, the relevant results are verified and simulated by using Maple software. Finally, the advantages and disadvantages of the above three methods listed in the paper are analyzed, and the conclusion was drawn by us. These methods are straightforward and concise in very easier ways.
\end{abstract}

\section{Introduction}

Since the 20th century, with the integration of various disciplines, people have gradually discovered nonlinear phenomena which exist in different disciplines but have common characteristics. The resulting nonlinear science is quickly and widely used in medical diagnosis, information technology, electrical engineering, and other fields, which greatly promotes the progress and development of human society [1].

Nonlinear science has laid a solid theoretical foundation for the safety, reliability, and stability of power systems [2-4]. By studying the nonlinear differential equations that appear in the ferromagnetic resonance overvoltage phenomenon, the safe operation of power grids at all levels can be effectively protected [5]. Aimed at the problem of asymmetric nonlinear oscillation in parallel operation of power stations, the harmonic linearization method is put forward, which is helpful to reasonably select the power grid structure and effectively improve the stability of power grid structure [6]. By constructing the nonlinear system model with random disturbance, the high-frequency faults of the power grid caused by disturbance can be greatly reduced $[4,7,8]$.
According to the nonlinear characteristics of the actual power system, a reliable and stable nonlinear power grid structure model is constructed, and the exact solution of the equation in the structure model is obtained, which plays a decisive role in the stable operation of the electrified wire netting $[9,10]$.

Study on the exact solutions of a wide variety of nonlinear equations can not only help people discover new nonlinear phenomena and their laws but also help ensure the reliability and stability of nonlinear calculations. Therefore, more and more scholars have proposed different methods for studying the exact solutions of a wide variety of nonlinear equations. Gardner et al. [11] used the backscattering argument of the Schrödinger equation to propose the inverse scattering transformation method. Wang and Li [12] used the principle of homogeneous balance to derive the nonlinear transformation of a great deal of nonlinear PDE and obtained the exact solution of the equation. Yu and Sun [13] presented a new $(3+1)$-dimensional KP-like nonlinear partial differential equation and construct the lump solutions, rationally localized in all directions in the space, to its two-dimensionally reduced cases. The method is based on a generalized bilinear 
differential equation, so we can build the lump solutions to the presented KP-like equation from special polynomial solutions to the aforementioned generalized bilinear equation which is helpful to improve the research efficiency. $\mathrm{Yu}$ and Sun [14] introduced the $(3+1)$-dimensional Kadomtsev-Petviashvili-Boussinesq-like equation. Based on this constructed bilinear Bäcklund transformation, some classes of exponential and rational traveling wave solutions with arbitrary wave numbers are presented. It provides a new idea for the follow-up research. Matinfar and Hosseini [15] formally studied the dynamic behavior of the solution obtained in the presence of linear and nonlinear effects. The research process is clear and interesting. Akinyemi et al. [16] studied singular solitons of the $(2+1)$-dimensional improved nonlinear Schrödinger equation with spatiotemporal dispersions, group velocity dispersions, and power law nonlinearity by using the Kudryashov method, a complex transformation as well as symbolic computations. The results show that the suggested approach is incredibly accurate and powerful and can be used to explore solitons and other forms of solutions of nonlinear wave models in applied sciences and engineering. In addition, there are many effective methods for solving exact solutions of nonlinear differential equations, for instance, Bäcklund transformation method [17], Painlevé truncation extension method [18, 19], continuation method [20], trial equation method [21], and Hirota bilinear method [22-25].

In 1976, Jaulent and Miodek [26] proposed the coupled Jaulent-Miodek equation when studying the relationship between the nonlinear evolution equation and the energydependent Schrödinger potential. In 2007, Feng and Li [27] used the theory of the plane dynamic system to study the existence of solitary waves and periodic waves of the coupled Jaulent-Miodek equation and obtained all possible explicit expressions. In 2009, Wazwaz [28] obtained multiple kink solutions and multiple singular kink solutions by studying the $(2+1)$-dimensional nonlinear evolution equation generated by the Jaulent-Miodek hierarchy. In 2013, Cai et al. [29] used the CK direct method to obtain new exact multiple kink and singular kink solutions through the symmetric transformation of the $(2+1)$-dimensional Jaulent-Miodek equation. In 2014, Ma et al. [30] used the auxiliary equation method and the Clarkson-Kruskal direct method to study $(2+1)$ dimensional nonlinear model systems and obtained some new exact solutions. In 2015, Matinfar et al. [31] studied some $(2+1)$-dimensional integrable models generated by the Jaulent-Miodek hierarchy and deduced the quasiperiodic solution of the $(2+1)$-dimensional integrable model. In 2016, Li et al. [32] obtained the classical Lie symmetry of the extended $(2+1)$-dimensional Jaulent-Miodek equation through the direct symmetry method and obtained a large number of new exact solutions by solving the reduced equation. In 2017, Wei et al. [33] studied the $(2+1)$-dimensional Jaulent-Miodek equation using the homogeneous balance method and obtained four new solitary wave solutions of the equation. In 2018, Motsepa et al. [34] derived the conservation law and obtained the traveling wave solutions of the $(2+1)$-dimensional Jaulent-Miodek equation. In 2018, Gu et al. [35] used the complex method to seek for the exact solutions of the $(2+1)$-dimensional Jaulent-Miodek equation. In 2019, Shehata and Zahran [36] used the JaulentMiodek equation to construct the exact traveling wave solutions of parametric equations with different methods. In 2020, Kaewta et al. [37] transformed the $(2+1)$-dimensional Jaulent-Miodek equation into a fourth-order partial differential equation and then obtained the exact solution which is simple and reliable.

The $(2+1)$-dimensional Jaulent-Miodek equation (see $[35,38,39])$ is considered:

$$
a_{1} u_{x t}+a_{2} u_{x}^{2} u_{x x}-u_{x x x x}-a_{3} u_{x x} u_{y}-a_{4} u_{x} u_{x y}+a_{5} u_{y y}=0 .
$$

In equation ( 1$), a_{i}$ is constant $(i=1,2, \cdots, 5$.)

Substituting traveling wave transform

$$
u(x, y, t)=v(z), \quad z=x+l y+\lambda t
$$

into equation (1) and integrating it deduce

$$
v^{\prime \prime \prime}-\left(a_{1} \lambda+a_{5} l^{2}\right) v^{\prime}+\frac{l b}{2}\left(v^{\prime}\right)^{2}-\frac{a_{2}}{3}\left(v^{\prime}\right)^{3}-\delta=0 .
$$

In equation (3), $b=a_{3}+a_{4}$, where $l, b$, and $\lambda$ are constants and $\delta$ is the integral constant. By setting $w=v^{\prime}$, equation (3) becomes

$$
w^{\prime \prime}-\left(a_{1} \lambda+a_{5} l^{2}\right) w+\frac{l b}{2} w^{2}-\frac{a_{2}}{3} w^{3}-\delta=0
$$

For the nonlinear $(2+1)$-dimensional Jaulent-Miodek equation, this paper adopts the $\exp (-\phi(z))$-expansion method, tanh method, and sine-cosine method to obtain the exact traveling wave solutions in turn.

\section{Introduction of the $\exp (-\phi(z))$-Expansion Method and Main Result}

Discuss a nonlinear partial differential equation (PDE) of the following form:

$$
P\left(\mu, \mu_{x}, \mu_{t}, \mu_{x x}, \mu_{x y}, \mu_{y y}, \mu_{y t}, \mu_{t t} \cdots\right)=0
$$

In equation (5), for the polynomial $P$ containing the nonlinear term, the highest order polynomial, and the unknown function $\mu(x, y, t)$, the following steps can be followed.

Step 1. By substituting traveling wave transform $\mu(x, y, t)=$ $w(z), \mathrm{z}=x+l y+\lambda t$ and putting them into equation (5), one can get the new equation, which is an ordinary differential equation $(\mathrm{ODE})$ :

$$
K\left(w, w^{\prime}, w^{\prime \prime}, w^{\prime \prime \prime}, \cdots\right)=0
$$


$K$ in equation (6) is both a polynomial of $w(z)$ and a polynomial of the derivatives.

Step 2. The traveling wave solutions of equation (6) are shown as indicated below:

$$
w(z)=\sum_{j=0}^{n} c_{j}(\exp (-\phi(z)))^{j} .
$$

Equation (7) contains a constant term $c_{j}(0 \leq j \leq n)$, and it will be discussed later; at the same time, $c_{j} \neq 0$ and $\phi=\phi(z)$ satisfy the ODE which will get

$$
\phi^{\prime}(z)=\exp (-\phi(z))+\mu \exp (\phi(z))+\delta_{1} .
$$

Next, different forms of equation (8) are shown under different conditions.

If $\delta_{1}^{2}-4 \mu>0, \mu \neq 0$, then

$\phi(z)=\ln \left(\frac{-\sqrt{\left(\delta_{1}^{2}-4 \mu\right)} \tanh \left(\left(\sqrt{\left(\delta_{1}^{2}-4 \mu\right)} / 2\right)(z+c)-\delta_{1}\right)}{2 \mu}\right)$,

$\phi(z)=\ln \left(\frac{-\sqrt{\left(\delta_{1}^{2}-4 \mu\right)} \operatorname{coth}\left(\left(\sqrt{\left(\delta_{1}^{2}-4 \mu\right)} / 2\right)(z+c)-\delta_{1}\right)}{2 \mu}\right)$.

If $\delta_{1}^{2}-4 \mu<0, \mu \neq 0$, then

$\phi(z)=\ln \left(\frac{-\sqrt{\left(4 \mu-\delta_{1}^{2}\right)} \tan \left(\left(\sqrt{\left(4 \mu-\delta_{1}^{2}\right)} / 2\right)(z+c)-\delta_{1}\right)}{2 \mu}\right)$,

$\phi(z)=\ln \left(\frac{-\sqrt{\left(4 \mu-\delta_{1}^{2}\right)} \cot \left(\left(\sqrt{\left(4 \mu-\delta_{1}^{2}\right) / 2}\right)(z+c)-\delta_{1}\right)}{2 \mu}\right)$.

If $\delta_{1}^{2}-4 \mu<0, \mu=0, \delta_{1} \neq 0$, then

$$
\phi(z)=-\ln \left(\frac{\delta_{1}}{\exp \left(\delta_{1}(z+c)-1\right)}\right) .
$$

If $\delta_{1}^{2}-4 \mu=0, \mu \neq 0, \delta_{1} \neq 0$, then

$$
\phi(z)=\ln \left(-\frac{2\left(\delta_{1}(z+c)+2\right)}{\delta_{1}^{2}(z+c)}\right) .
$$

$$
\text { If } \delta_{1}^{2}-4 \mu=0, \mu=0, \delta_{1}=0 \text {, then }
$$

$$
\phi(z)=\ln (z+c) .
$$

In the above equations, $c_{n} \neq 0, \delta_{1}$, and $\mu$ are undetermined constants, and the constant $c$ can take any value. We consider the homogeneous balance between nonlinear terms and highest order derivatives of equation (6), and then, we can get the positive integer $n$.

Step 3. Through simultaneous equations (6) and (7), the polynomial about $\exp (-\phi(z))$ can be obtained. Calculating all the coefficients of the same power of $\exp (-\phi(z))$ to zero, we can get a set of simultaneous equations.

Theorem 1. Based on the $\exp (-\phi(z))$-expansion method, we can get fourteen kinds of solutions to equation (3).

If $\delta_{1}^{2}-4 \mu>0, \mu \neq 0$,

$$
\begin{aligned}
v_{11}= & \frac{l b+\sqrt{6 a_{2}} \delta_{1}}{2 a_{2}} z+\frac{2 \sqrt{\left(6 / a_{2}\right)} \mu}{\delta_{1}^{2}-4 \mu} \\
& \cdot \ln \frac{\left(\tanh ^{2}\left(\left(\sqrt{\delta_{1}^{2}-4 \mu}(z+c) / 2\right)-\delta_{1}\right)-1\right)}{\tanh ^{2}\left(\left(\sqrt{\delta_{1}^{2}-4 \mu}(z+c) / 2\right)-\delta_{1}\right)}+d_{1},
\end{aligned}
$$

$$
\begin{aligned}
v_{12}= & \frac{l b+\sqrt{6 a_{2}} \delta_{1}}{2 a_{2}} z+\frac{2 \sqrt{\left(6 / a_{2}\right)} \mu}{\delta_{1}^{2}-4 \mu} \\
& \cdot \ln \frac{\left(\operatorname{coth}^{2}\left(\left(\sqrt{\delta_{1}^{2}-4 \mu}(z+c) / 2\right)-\delta_{1}\right)-1\right)}{\operatorname{coth}^{2}\left(\left(\sqrt{\delta_{1}^{2}-4 \mu}(z+c) / 2\right)-\delta_{1}\right)}+d_{2},
\end{aligned}
$$

$$
\begin{aligned}
v_{21}= & \frac{l b-\sqrt{6 a_{2}} \delta_{1}}{2 a_{2}} z+\frac{2 \sqrt{\left(6 / a_{2}\right)} \mu}{\delta_{1}^{2}-4 \mu} \\
& \cdot \ln \frac{\tanh ^{2}\left(\left(\sqrt{\delta_{1}^{2}-4 \mu}(z+c) / 2\right)-\delta_{1}\right)}{\left(\tanh ^{2}\left(\left(\sqrt{\delta_{1}^{2}-4 \mu}(z+c) / 2\right)-\delta_{1}\right)-1\right)}+d_{3},
\end{aligned}
$$

$$
\begin{aligned}
v_{22}= & \frac{l b-\sqrt{6 a_{2}} \delta_{1}}{2 a_{2}} z+\frac{2 \sqrt{\left(6 / a_{2}\right)} \mu}{\delta_{1}^{2}-4 \mu} \\
& \cdot \ln \frac{\operatorname{coth}^{2}\left(\left(\sqrt{\delta_{1}^{2}-4 \mu}(z+c) / 2\right)-\delta_{1}\right)}{\left(\operatorname{coth}^{2}\left(\left(\sqrt{\delta_{1}^{2}-4 \mu}(z+c) / 2\right)-\delta_{1}\right)-1\right)}+d_{4},
\end{aligned}
$$


where $\mu=\left(\left(4 a_{5} l^{2}+4 a_{1} \lambda+2 \delta_{1}^{2}\right) a_{2}-l^{2} b^{2}\right) / 8 a_{2}, \quad \delta=\left(l^{3} b^{3}-\right.$ $\left.6 a_{2} l b\left(a_{5} l^{2}+a_{1} \lambda\right)\right) / 12 a_{2}^{2}$, and $d_{1}, d_{2}, d_{3}$, and $d_{4}$ are integral constants.

$$
\begin{aligned}
& \text { If } \delta_{1}^{2}-4 \mu<0, \mu \neq 0, \\
& v_{13}=\frac{l b+\sqrt{6 a_{2}} \delta_{1}}{2 a_{2}} z+\frac{2 \sqrt{\left(6 / a_{2}\right)} \mu}{4 \mu-\delta_{1}^{2}} \\
& \cdot \ln \frac{\left(\tan \left(\left(\sqrt{4 \mu-\delta_{1}^{2}}(z+c) / 2\right)-\delta_{1}\right)^{2}+1\right)}{\tan ^{2}\left(\left(\sqrt{4 \mu-\delta_{1}^{2}}(z+c) / 2\right)-\delta_{1}\right)}+d_{5},
\end{aligned}
$$

$$
\begin{aligned}
v_{14}= & \frac{l b+\sqrt{6 a_{2}} \delta_{1}}{2 a_{2}} z+\frac{2 \sqrt{\left(6 / a_{2}\right)} \mu}{4 \mu-\delta_{1}^{2}} \\
& \cdot \ln \frac{\cot ^{2}\left(\left(\sqrt{4 \mu-\delta_{1}^{2}}(z+c) / 2\right)-\delta_{1}\right)}{\left(\cot \left(\left(\sqrt{4 \mu-\delta_{1}^{2}}(z+c) / 2\right)-\delta_{1}\right)^{2}+1\right)}+d_{6},
\end{aligned}
$$

$$
\begin{aligned}
v_{23}= & \frac{l b-\sqrt{6 a_{2}} \delta_{1}}{2 a_{2}} z+\frac{2 \sqrt{\left(6 / a_{2}\right)} \mu}{4 \mu-\delta_{1}^{2}} \\
& \cdot \ln \frac{\tan ^{2}\left(\left(\sqrt{4 \mu-\delta_{1}^{2}}(z+c) / 2\right)-\delta_{1}\right)}{\left(\tan \left(\left(\sqrt{4 \mu-\delta_{1}^{2}}(z+c) / 2\right)-\delta_{1}\right)^{2}+1\right)}+d_{7},
\end{aligned}
$$

$$
\begin{aligned}
v_{24}= & \frac{l b-\sqrt{6 a_{2}} \delta_{1}}{2 a_{2}} z+\frac{2 \sqrt{\left(6 / a_{2}\right)} \mu}{4 \mu-\delta_{1}^{2}} \\
& \cdot \ln \frac{\left(\cot \left(\left(\sqrt{4 \mu-\delta_{1}^{2}}(z+c) / 2\right)-\delta_{1}\right)^{2}+1\right)}{\cot ^{2}\left(\left(\sqrt{4 \mu-\delta_{1}^{2}}(z+c) / 2\right)-\delta_{1}\right)}+d_{8},
\end{aligned}
$$

where $\mu=\left(\left(4 a_{5} l^{2}+4 a_{1} \lambda+2 \delta_{1}^{2}\right) a_{2}-l^{2} b^{2}\right) / 8 a_{2}, \quad \delta=\left(l^{3} b^{3}-\right.$ $\left.6 a_{2} l b\left(a_{5} l^{2}+a_{1} \lambda\right)\right) / 12 a_{2}^{2}$, and $d_{5}, d_{6}, d_{7}$, and $d_{8}$ are integral constants.

$$
\begin{aligned}
& \text { If } \begin{aligned}
\delta_{1}^{2}-4 \mu & <0, \mu=0, \delta_{1} \neq 0 \\
v_{15} & =\frac{l b+\sqrt{6 a_{2}} \delta_{1}}{2 a_{2}} z-\frac{\sqrt{6 / a_{2}}}{e^{\delta_{1}(z+c)-1}}+d_{9}, \\
v_{25} & =\frac{l b-\sqrt{6 a_{2}} \delta_{1}}{2 a_{2}} z+\frac{\sqrt{6 / a_{2}}}{e^{\delta_{1}(z+c)-1}}+d_{10}
\end{aligned}
\end{aligned}
$$

where $\mu=0, \delta=\left(-(1 / 2) l^{3} b^{3} \mp 3 \sqrt{6} a_{2}^{3 / 2} \delta_{1}^{3}\right) / 12 a_{2}^{2}$, and $d_{9}$ and $d_{10}$ are integral constants.

$$
\text { If } \delta_{1}^{2}-4 \mu=0, \mu \neq 0, \delta_{1} \neq 0 \text {, }
$$

$$
\begin{aligned}
& v_{16}=\frac{l b}{2 a_{2}} z+\sqrt{\frac{6}{a_{2}}} \ln \left(\delta_{1}(z+c)+2\right)+d_{11}, \\
& v_{26}=\frac{l b}{2 a_{2}} z-\sqrt{\frac{6}{a_{2}}} \ln \left(\delta_{1}(z+c)+2\right)+d_{12},
\end{aligned}
$$

where $\mu=(1 / 4) \delta_{1}^{2}, \delta=-l^{3} b^{3} / 24 a_{2}^{2}$, and $d_{11}$ and $d_{12}$ are integral constants.

$$
\text { If } \begin{aligned}
\delta_{1}^{2}-4 \mu & =0, \mu=0, \delta_{1}=0, \\
v_{17} & =\frac{l b}{2 a_{2}} z+\sqrt{\frac{6}{a_{2}}} \ln (z+c)+d_{13}, \\
v_{27} & =\frac{l b}{2 a_{2}} z-\sqrt{\frac{6}{a_{2}}} \ln (z+c)+d_{14},
\end{aligned}
$$

where $\mu=0, \delta=-l^{3} b^{3} / 24 a_{2}^{2}$, and $d_{13}$ and $d_{14}$ are integral constants.

\section{Introduction of the tanh Method and Main Result}

The canonical tanh method proposed by $[40,41]$ includes the following steps.

Step 1. The tanh method takes the form of introducing a new independent variable:

$$
Y=\tanh (\mu z) .
$$

The derivatives of the independent variable change as follows:

$$
\begin{aligned}
\frac{d}{d z} & =\mu\left(1-Y^{2}\right) \frac{d}{d Y}, \\
\frac{d^{2}}{d z^{2}} & =\mu^{2}\left(1-Y^{2}\right)\left(-2 Y \frac{d}{d Y}+\left(1-Y^{2}\right) \frac{d^{2}}{d Y^{2}}\right),
\end{aligned}
$$

where other derivatives of this kind can be obtained in a similar way.

Step 2. The premise of using the tanh method is to assume that the solution $w(z)$ has the form

$$
w(z)=\sum_{m=0}^{M} b_{m} Y^{m},
$$

where $M$ is a positive integer and its value needs to be determined here. Then, by substituting equations (31) and (32) 
into the simplified ODE, we can get equations about the $Y$ powers.

Step 3. Through balancing the highest order of linear and nonlinear terms, we can get parameter $M$. By equating the power coefficients of $Y$ in the obtained equations, a set of algebraic equations about $b_{m}, \mu$, and $c$ can be obtained. After the above parameters are determined, an analytical solution can be obtained by using equation (33) under closed condition.

Theorem 2. By employing the tanh method, we found that there will be two forms of solutions of (3).

$$
\begin{aligned}
v_{T 1}= & \frac{l b}{2 a_{2}} z-\sqrt{\frac{3}{2 a_{2}}} \\
& \cdot \ln \left(-\operatorname{sech}\left(\frac{1}{2} \sqrt{\frac{l^{2} b^{2}-4 a_{2}\left(a_{5} l^{2}+a_{1} \lambda\right)}{2 a_{2}}} z\right)^{2}\right)+d_{15},
\end{aligned}
$$

$$
\begin{aligned}
v_{T 2}= & \frac{l b}{2 a_{2}} z+\sqrt{\frac{3}{2 a_{2}}} \\
& \cdot \ln \left(-\operatorname{sech}\left(\frac{1}{2} \sqrt{\frac{l^{2} b^{2}-4 a_{2}\left(a_{5} l^{2}+a_{1} \lambda\right)}{2 a_{2}} z}\right)^{2}\right)+d_{16},
\end{aligned}
$$

where $d_{15}$ and $d_{16}$ are integral constants.

\section{Introduction of the Sine-Cosine Method and Main Result}

It is proven that the method of sine-cosine is effective in dealing with compaction problems. Next, the report describes this method briefly below, as the details have been shown in [42].

The sine-cosine method applies to the following form:

$$
u(x, t)=\left\{\lambda_{1} \cos ^{\beta_{1}}(\mu z)\right\}, \quad|z|<\frac{\pi}{2 \mu},
$$

or another form

$$
u(x, t)=\left\{\lambda_{1} \sin ^{\beta_{1}}(\mu z)\right\}, \quad|z|<\frac{\pi}{\mu}
$$

otherwise, it is zero. The values of $\lambda_{1}, \mu$, and $\beta_{1}$ parameters were measured. In equation (5), for the polynomial $P$ containing the nonlinear term, the highest order polynomial, and the unknown function $\mu(x, y, t)$, the following steps can be followed.

Step 1. By substituting traveling wave transform $\mu(x, y, t)=$ $w(z), z=x+l y+\lambda t$ into equation (5), it is transformed into ordinary differential equation (6). $K$ in equation (6) is both a polynomial of $w(z)$ and a polynomial of the derivatives.

Step 2. The traveling wave solutions of equation (6) are shown as indicated below:

$$
w(z)=\left\{\lambda_{1} \cos ^{\beta_{1}}(\mu z)\right\}, \quad|z|<\frac{\pi}{2 \mu},
$$

or another form

$$
w(z)=\left\{\lambda_{1} \sin ^{\beta_{1}}(\mu z)\right\}, \quad|z|<\frac{\pi}{\mu}
$$

otherwise, it is zero. The values of $\lambda_{1}, \mu$, and $\beta_{1}$ parameters were measured.

Step 3. For the cosine or sine functions of the same homomorphic power, extract its undetermined coefficients and set them to zero, so we have simultaneous equations. The determination of the solutions of equation (5) can be obtained by obtaining the values of $\lambda_{1}, \mu$, and $\beta_{1}$.

Theorem 3. Based on the sine-cosine method, the four forms of all meromorphic solutions $v$ of equation (3) are shown as indicated below.

(1) The cosine method is used to solve equation (3) as follows:

$$
\begin{aligned}
v_{c 1}(z)= & \sqrt{\frac{6}{a_{2}}} \ln \left(\sec \left(\sqrt{-a_{5} l^{2}-a_{1} \lambda z}\right)\right. \\
& \left.+\tan \left(\sqrt{-a_{5} l^{2}-a_{1} \lambda z}\right)\right)+d_{17}, \\
v_{c 2}(z)= & -\sqrt{\frac{6}{a_{2}}} \ln \left(\sec \left(\sqrt{-a_{5} l^{2}-a_{1} \lambda z}\right)\right. \\
& \left.+\tan \left(\sqrt{-a_{5} l^{2}-a_{1} \lambda z}\right)\right)+d_{18},
\end{aligned}
$$

where $d_{17}$ and $d_{18}$ are integral constants.

(2) The sine method is used to solve equation (3) as follows:

$$
\begin{aligned}
v_{s 1}(z)= & -\sqrt{\frac{6}{a_{2}}} \ln \left(\csc \left(\sqrt{-a_{5} l^{2}-a_{1} \lambda z}\right)\right. \\
& \left.+\cot \left(\sqrt{-a_{5} l^{2}-a_{1} \lambda z}\right)\right)+d_{19} \\
v_{s 2}(z)= & \sqrt{\frac{6}{a_{2}}} \ln \left(\csc \left(\sqrt{-a_{5} l^{2}-a_{1} \lambda z}\right)\right. \\
& \left.+\cot \left(\sqrt{-a_{5} l^{2}-a_{1} \lambda z}\right)\right)+d_{20}
\end{aligned}
$$

where $d_{19}$ and $d_{20}$ are integral constants. 


\section{Proof of Theorems}

5.1. Proof of Theorem 1. By homogeneous equilibrium of $w^{\prime \prime}$ and $w^{3}$ in equation (4), we can get

$$
w(z)=\mathrm{c}_{0}+\mathrm{c}_{1} \exp (-\phi(z)) .
$$

In the above formula, $c_{1} \neq 0$ and $c_{0}$ are undetermined constants. Moreover, $\phi(z)$ satisfies equation (8), where the constants $\delta_{1}$ and $\mu$ can be arbitrarily taken.

From equation (42), we insert $w, w^{3}$, and $w^{\prime \prime}$ into equation (4). By calculating all the coefficients of the same power of $\exp (-\phi(z))$ to zero, the following equations can be obtained:

$$
\left\{\begin{array}{l}
e^{-3 \phi(z)} \text { coefficient : } 2 c_{1}-\frac{a_{2} c_{1}^{3}}{3}=0, \\
e^{-2 \phi(z)} \text { coefficient : } 3 c_{1} \delta_{1}+\frac{l b c_{1}^{2}}{2}-a_{2} c_{0} c_{1}^{2}=0, \\
e^{-1 \phi(z)} \text { coefficient : } 2 c_{1} \mu+c_{1} \delta_{1}^{2}-a_{1} c_{1} \lambda-a_{5} l^{2} c_{1}+l b c_{0} c_{1}-a_{2} c_{0}^{2} c_{1}=0, \\
e^{-0 \phi(z)} \text { coefficient : } c_{1} \delta_{1} \mu-a_{1} c_{0} \lambda-a_{5} c_{0} l^{2}+\frac{l b c_{0}^{2}}{2}-\frac{a_{2} c_{0}^{3}}{3}-\delta=0 .
\end{array}\right.
$$

Simultaneously, the above equations can be obtained:

$$
\begin{aligned}
& \left\{\begin{array}{l}
c_{0}=\frac{l b+\sqrt{6 a_{2}} \delta_{1}}{2 a_{2}}, \\
c_{1}=\sqrt{\frac{6}{a_{2}}}
\end{array}\right. \\
& \text { or }\left\{\begin{array}{l}
c_{0}=\frac{l b-\sqrt{6 a_{2}} \delta_{1}}{2 a_{2}}, \\
c_{1}=-\sqrt{\frac{6}{a_{2}} .}
\end{array}\right.
\end{aligned}
$$

Substitute the result of the above formula into (42) to get

$$
\begin{array}{r}
w_{1}(z)=\frac{l b+\sqrt{6 a_{2}} \delta_{1}}{2 a_{2}}+\sqrt{\frac{6}{a_{2}}} e^{-\phi(z)}, \\
\text { or } w_{2}(z)=\frac{l b-\sqrt{6 a_{2}} \delta_{1}}{2 a_{2}}-\sqrt{\frac{6}{a_{2}}} e^{-\phi(z)} .
\end{array}
$$

We apply equations (9)-(15) into equations (45) and (46), respectively; the traveling wave solutions of the $(2+1)$ dimensional Jaulent-Miodek equations are shown as indicated below.

$$
\begin{aligned}
& \text { If } \delta_{1}^{2}-4 \mu>0, \mu \neq 0, \\
& w_{11}=\frac{l b+\sqrt{6 a_{2}} \delta_{1}}{2 a_{2}} \\
& -\frac{2 \sqrt{\left(6 / a_{2}\right)} \mu}{\sqrt{\delta_{1}^{2}-4 \mu} \tanh \left(\left((z+c) \sqrt{\delta_{1}^{2}-4 \mu / 2}\right)-\delta_{1}\right)},
\end{aligned}
$$

$$
\begin{aligned}
& v_{11}= \int w_{11} d z=\frac{l b+\sqrt{6 a_{2}} \delta_{1}}{2 a_{2}} z+\frac{2 \sqrt{\left(6 / a_{2}\right)} \mu}{\delta_{1}^{2}-4 \mu} \\
& \cdot \ln \frac{\left(\tanh ^{2}\left(\left(\sqrt{\delta_{1}^{2}-4 \mu}(z+c) / 2\right)-\delta_{1}\right)-1\right)}{\tanh ^{2}\left(\left(\sqrt{\delta_{1}^{2}-4 \mu}(z+c) / 2\right)-\delta_{1}\right)}+d_{1},
\end{aligned}
$$

$$
\begin{aligned}
w_{12}= & \frac{l b+\sqrt{6 a_{2}} \delta_{1}}{2 a_{2}} \\
& -\frac{2 \sqrt{\left(6 / a_{2}\right)} \mu}{\sqrt{\delta_{1}^{2}-4 \mu} \operatorname{coth}\left(\left((z+c) \sqrt{\delta_{1}^{2}-4 \mu / 2}\right)-\delta_{1}\right)}, \\
v_{12}= & \int w_{12} d z=\frac{l b+\sqrt{6 a_{2}} \delta_{1}}{2 a_{2}}+\frac{2 \sqrt{\left(6 / a_{2}\right)} \mu}{\delta_{1}^{2}-4 \mu} \\
& \cdot \ln \frac{\left(\operatorname{coth}^{2}\left(\left(\sqrt{\delta_{1}^{2}-4 \mu}(z+c) / 2\right)-\delta_{1}\right)-1\right)}{\operatorname{coth}^{2}\left(\left(\sqrt{\delta_{1}^{2}-4 \mu}(z+c) / 2\right)-\delta_{1}\right)}+d_{2},
\end{aligned}
$$

$$
\begin{aligned}
w_{21}= & \frac{l b-\sqrt{6 a_{2}} \delta_{1}}{2 a_{2}} \\
& +\frac{2 \sqrt{\left(6 / a_{2}\right)} \mu}{\sqrt{\delta_{1}^{2}-4 \mu} \tanh \left(\left((z+c) \sqrt{\left.\left.\delta_{1}^{2}-4 \mu / 2\right)-\delta_{1}\right)}\right.\right.}, \\
v_{21}= & \int w_{21} d z=\frac{l b-\sqrt{6 a_{2}} \delta_{1} z+\frac{2 \sqrt{\left(6 / a_{2}\right)} \mu}{2 a_{2}}}{\delta_{1}^{2}-4 \mu} \\
& \cdot \ln \frac{\tanh ^{2}\left(\left(\sqrt{\delta_{1}^{2}-4 \mu}(z+c) / 2\right)-\delta_{1}\right)}{\left(\tanh ^{2}\left(\left(\sqrt{\delta_{1}^{2}-4 \mu}(z+c) / 2\right)-\delta_{1}\right)-1\right)}
\end{aligned}
$$$$
+d_{3}
$$

$$
\begin{aligned}
w_{22}= & \frac{l b-\sqrt{6 a_{2}} \delta_{1}}{2 a_{2}} \\
& +\frac{2 \sqrt{\left(6 / a_{2}\right)} \mu}{\sqrt{\delta_{1}^{2}-4 \mu} \operatorname{coth}\left(\left((z+c) \sqrt{\delta_{1}^{2}-4 \mu / 2}\right)-\delta_{1}\right)},
\end{aligned}
$$$$
v_{22}=\int w_{22} d z=\frac{l b-\sqrt{6 a_{2}} \delta_{1}}{2 a_{2}} z+\frac{2 \sqrt{\left(6 / a_{2}\right)} \mu}{\delta_{1}^{2}-4 \mu}
$$$$
\cdot \ln \frac{\operatorname{coth}^{2}\left(\left(\sqrt{\delta_{1}^{2}-4 \mu}(z+c) / 2\right)-\delta_{1}\right)}{\left(\operatorname{coth}^{2}\left(\left(\sqrt{\delta_{1}^{2}-4 \mu}(z+c) / 2\right)-\delta_{1}\right)-1\right)}+d_{4}
$$ 
where $\mu=\left(\left(4 a_{5} l^{2}+4 a_{1} \lambda+2 \delta_{1}^{2}\right) a_{2}-l^{2} b^{2}\right) / 8 a_{2}, \delta=\left(l^{3} b^{3}-6\right.$ $\left.a_{2} l b\left(a_{5} l^{2}+a_{1} \lambda\right)\right) / 12 a_{2}^{2}$, and $d_{1}, d_{2}, d_{3}$, and $d_{4}$ are integral constants.

$$
\begin{aligned}
& \text { If } \delta_{1}^{2}-4 \mu<0, \mu \neq 0, \\
& w_{13}=\frac{l b+\sqrt{6 a_{2}} \delta_{1}}{2 a_{2}} \\
& -\frac{2 \sqrt{\left(6 / a_{2}\right)} \mu}{\sqrt{4 \mu-\delta_{1}^{2}} \tan \left(\left((z+c) \sqrt{4 \mu-\delta_{1}^{2}} / 2\right)-\delta_{1}\right)}, \\
& v_{13}=\int w_{13} d z=\frac{l b+\sqrt{6 a_{2}} \delta_{1}}{2 a_{2}} z+\frac{2 \sqrt{\left(6 / a_{2}\right)} \mu}{4 \mu-\delta_{1}^{2}} \\
& \cdot \ln \frac{\left(\tan \left(\left(\sqrt{4 \mu-\delta_{1}^{2}}(z+c) / 2\right)-\delta_{1}\right)^{2}+1\right)}{\tan ^{2}\left(\left(\sqrt{4 \mu-\delta_{1}^{2}}(z+c) / 2\right)-\delta_{1}\right)}+d_{5},
\end{aligned}
$$

$$
\begin{aligned}
w_{14}= & \frac{l b+\sqrt{6 a_{2}} \delta_{1}}{2 a_{2}} \\
& -\frac{2 \sqrt{\left(6 / a_{2}\right)} \mu}{\sqrt{4 \mu-\delta_{1}^{2}} \cot \left(\left((z+c) \sqrt{4 \mu-\delta_{1}^{2}} / 2\right)-\delta_{1}\right)}, \\
v_{14}= & \int w_{14} d z=\frac{l b+\sqrt{6 a_{2}} \delta_{1}}{2 a_{2}} z+\frac{2 \sqrt{6 / a_{2}} \mu}{4 \mu-\delta_{1}^{2}} \\
& \cdot \ln \frac{\cot ^{2}\left(\left(\sqrt{4 \mu-\delta_{1}^{2}}(z+c) / 2\right)-\delta_{1}\right)}{\left(\cot \left(\left(\sqrt{4 \mu-\delta_{1}^{2}}(z+c) / 2\right)-\delta_{1}\right)^{2}+1\right)}+d_{6},
\end{aligned}
$$

$$
\begin{aligned}
w_{23}= & \frac{l b-\sqrt{6 a_{2}} \delta_{1}}{2 a_{2}} \\
& +\frac{2 \sqrt{\left(6 / a_{2}\right)} \mu}{\sqrt{4 \mu-\delta_{1}^{2}} \tan \left(\left((z+c) \sqrt{4 \mu-\delta_{1}^{2}} / 2\right)-\delta_{1}\right)}, \\
v_{23}= & \int w_{23} d z=\frac{l b-\sqrt{6 a_{2}} \delta_{1}}{2 a_{2}} z+\frac{2 \sqrt{\left(6 / a_{2}\right)} \mu}{4 \mu-\delta_{1}^{2}} \\
& \cdot \ln \frac{\tan ^{2}\left(\left(\sqrt{4 \mu-\delta_{1}^{2}}(z+c) / 2\right)-\delta_{1}\right)}{\left(\tan \left(\left(\sqrt{4 \mu-\delta_{1}^{2}}(z+c) / 2\right)-\delta_{1}\right)^{2}+1\right)}+d_{7},
\end{aligned}
$$

$$
\begin{aligned}
w_{24}= & \frac{l b-\sqrt{6 a_{2}} \delta_{1}}{2 a_{2}} \\
& +\frac{2 \sqrt{\left(6 / a_{2}\right)} \mu}{\sqrt{4 \mu-\delta_{1}^{2}} \cot \left(\left((z+c) \sqrt{4 \mu-\delta_{1}^{2}} / 2\right)-\delta_{1}\right)}, \\
v_{24}= & \int w_{24} d z=\frac{l b-\sqrt{6 a_{2}} \delta_{1}}{2 a_{2}} z+\frac{2 \sqrt{\left(6 / a_{2}\right)} \mu}{4 \mu-\delta_{1}^{2}} \\
& \cdot \ln \frac{\left(\cot \left(\left(\sqrt{4 \mu-\delta_{1}^{2}}(z+c) / 2\right)-\delta_{1}\right)^{2}+1\right)}{\cot ^{2}\left(\left(\sqrt{4 \mu-\delta_{1}^{2}}(z+c) / 2\right)-\delta_{1}\right)}+d_{8},
\end{aligned}
$$

where $\mu=\left(\left(4 a_{5} l^{2}+4 a_{1} \lambda+2 \delta_{1}^{2}\right) a_{2}-l^{2} b^{2}\right) / 8 a_{2}, \quad \delta=\left(l^{3} b^{3}-\right.$ $\left.6 a_{2} l b\left(a_{5} l^{2}+a_{1} \lambda\right)\right) / 12 a_{2}^{2}$, and $d_{5}, d_{6}, d_{7}$, and $d_{8}$ are integral constants.

$$
\begin{aligned}
& \text { If } \delta_{1}^{2}-4 \mu<0, \mu=0, \delta_{1} \neq 0, \\
& w_{15}=\frac{l b+\sqrt{6 a_{2}} \delta_{1}}{2 a_{2}}+\frac{\sqrt{\left(6 / a_{2}\right)} \delta_{1}}{e^{\delta_{1}(z+c)-1}}, \\
& v_{15}=\int w_{15} d z=\frac{l b+\sqrt{6 a_{2}} \delta_{1}}{2 a_{2}} z-\frac{\sqrt{6 / a_{2}}}{e^{\delta_{1}(z+c)-1}}+d_{9}, \\
& w_{25}=\frac{l b-\sqrt{6 a_{2}} \delta_{1}}{2 a_{2}}-\frac{\sqrt{\left(6 / a_{2}\right)} \delta_{1}}{e^{\delta_{1}(z+c)-1}}, \\
& v_{25}=\int w_{25} d z=\frac{l b-\sqrt{6 a_{2}} \delta_{1}}{2 a_{2}} z+\frac{\sqrt{6 / a_{2}}}{e^{\delta_{1}(z+c)-1}}+d_{10},
\end{aligned}
$$

where $\mu=0, \delta=\left(-(1 / 2) l^{3} b^{3} \mp 3 \sqrt{6} a_{2}^{3 / 2} \delta_{1}^{3}\right) / 12 a_{2}^{2}$, and $d_{9}$ and $d_{10}$ are integral constants.

If $\delta_{1}^{2}-4 \mu=0, \mu \neq 0, \delta_{1} \neq 0$,

$$
\begin{aligned}
& w_{16}=\frac{l b+\sqrt{6 a_{2}} \delta_{1}}{2 a_{2}}-\frac{\sqrt{\left(6 / a_{2}\right)} \delta_{1}^{2}(z+c)}{2 \delta_{1}(z+c)+4}, \\
& v_{16}=\int w_{16} d z=\frac{l b}{2 a_{2}} z+\sqrt{\frac{6}{a_{2}}} \ln \left(\delta_{1}(z+c)+2\right)+d_{11}, \\
& w_{26}=\frac{l b-\sqrt{6 a_{2}} \delta_{1}}{2 a_{2}}+\frac{\sqrt{\left(6 / a_{2}\right.} \delta_{1}^{2}(z+c)}{2 \delta_{1}(z+c)+4}, \\
& v_{26}=\int w_{26} d z=\frac{l b}{2 a_{2}} z-\sqrt{\frac{6}{a_{2}}} \ln \left(\delta_{1}(z+c)+2\right)+d_{12},
\end{aligned}
$$

where $\mu=(1 / 4) \delta_{1}^{2}, \delta=-l^{3} b^{3} / 24 a_{2}^{2}$, and $d_{11}$ and $d_{12}$ are integral constants. 


$$
\begin{aligned}
& \text { If } \delta_{1}^{2}-4 \mu=0, \mu=0, \delta_{1}=0, \\
& \begin{array}{l}
w_{17}=\frac{l b}{2 a_{2}}+\frac{\sqrt{6 / a_{2}}}{(z+c)}, \\
v_{17}=\int w_{17} d z=\frac{l b}{2 a_{2}} z+\sqrt{\frac{6}{a_{2}}} \ln (z+c)+d_{13}, \\
w_{27}=\frac{l b}{2 a_{2}}-\frac{\sqrt{6 / a_{2}}}{(z+c)}, \\
v_{27}=\int w_{27} d z=\frac{l b}{2 a_{2}} z-\sqrt{\frac{6}{a_{2}}} \ln (z+c)+d_{14},
\end{array}
\end{aligned}
$$

where $\mu=0, \delta=-l^{3} b^{3} / 24 a_{2}^{2}$, and $d_{13}$ and $d_{14}$ are integral constants.

5.2. Proof of Theorem 2. The tanh method takes the form of introducing a new independent variable:

$$
Y=\tanh (\mu z)
$$
follows:

The derivatives of the independent variable change as

$$
\begin{aligned}
\frac{d}{d z} & =\mu\left(1-Y^{2}\right) \frac{d}{d Y} \\
\frac{d^{2}}{d z^{2}} & =\mu^{2}\left(1-Y^{2}\right)\left(-2 Y \frac{d}{d Y}+\left(1-Y^{2}\right) \frac{d^{2}}{d Y^{2}}\right) .
\end{aligned}
$$

For the nonlinear $(2+1)$-dimensional Jaulent-Miodek equation,

$w^{\prime \prime}-\left(a_{1} \lambda+a_{5} l^{2}\right) w+\frac{l b}{2} w^{2}-\frac{a_{2}}{3} w^{3}-\delta=0, w=\sum_{m=0}^{M} b_{m} Y^{m}$

Balancing the highest order of linear and nonlinear terms, we get

$$
3 M=M+2 .
$$

It is derived that $M=1$; therefore,

$$
w(z)=b_{0}+b_{1} Y=b_{0}+b_{1} \tanh (\mu z)
$$

In the above formula,

$$
\begin{aligned}
& \frac{d w}{d Y}=b_{1}, \\
& \frac{d^{2} w}{d Y^{2}}=0,
\end{aligned}
$$

where $b_{0}, b_{1}$, and $\mu$ appearing in the above are all undetermined constants; substituting (62) and (63) into (64), we get

$$
\begin{aligned}
& \mu^{2}\left(1-Y^{2}\right)\left(-2 Y w^{\prime}+\left(1-Y^{2}\right) w^{\prime \prime}\right) \\
& \quad-\left(a_{1} \lambda+a_{5} l^{2}\right) w+\frac{l b}{2} w^{2}-\frac{a_{2}}{3} w^{3}-\delta=0 .
\end{aligned}
$$

Substituting (66) and (67) into (68), we get

$$
\begin{gathered}
\mu^{2}\left(1-Y^{2}\right)\left(-2 Y b_{1}\right)-\left(a_{1} \lambda+a_{5} l^{2}\right)\left(b_{0}+b_{1} Y\right) \\
+\frac{l b}{2}\left(b_{0}+b_{1} Y\right)^{2}-\frac{a_{2}}{3}\left(b_{0}+b_{1} Y\right)^{3}-\delta=0 .
\end{gathered}
$$

In equation (69), $a_{1}, a_{5}, l, b$, and $\lambda$ are all constants and $\delta$ is the integral constant. Extract the coefficients before all the powers of $Y$, and set each coefficient to zero, so as to obtain the simultaneous equations about the undetermined coefficients $b_{0}, b_{1}$, and $\mu$ :

$$
\left\{\begin{array}{l}
Y^{3} \text { coefficient }: 2 b_{1} \mu^{2}-\frac{a_{2} b_{1}^{3}}{3}=0, \\
Y^{2} \text { coefficient }: \frac{l b b_{1}^{2}}{2}-a_{2} b_{0} b_{1}^{2}=0, \\
Y^{1} \text { coefficient }: l b b_{0} b_{1}-a_{5} b_{1} l^{2}-a_{1} b_{1} \lambda-2 b_{1} \mu^{2}-a_{2} b_{0}^{2} b_{1}=0, \\
Y^{0} \text { coefficient }: \frac{l b b_{0}^{2}}{2}-a_{1} b_{0} \lambda-a_{5} b_{0} 1^{2}-\frac{a_{2} b_{0}^{3}}{3}-\delta=0 .
\end{array}\right.
$$

Solving the above simultaneous equations can get

$$
\begin{aligned}
& b_{0}=\frac{l b}{2 a_{2}}, \\
& b_{1}= \pm \sqrt{\frac{3(l b)^{2}-12 a_{2}\left(a_{1} \lambda+a_{5} l^{2}\right)}{4 a_{2}^{2}}}, \\
& \mu= \pm \sqrt{\frac{(l b)^{2}-4 a_{2}\left(a_{1} \lambda+a_{5} l^{2}\right)}{8 a_{2}}}, \\
& \delta=\frac{(l b)^{3}-6 a_{2} l b\left(a_{1} \lambda+a_{5} l^{2}\right)}{12 a_{2}^{2}} .
\end{aligned}
$$

Substituting $b_{0}, b_{1}$, and $\mu$ into equation (68), we can get solutions for two cases:

$$
\begin{aligned}
w_{T 1}= & \frac{l b}{2 a_{2}}+\sqrt{\frac{3(l b)^{2}-12 a_{2}\left(a_{1} \lambda+a_{5} l^{2}\right)}{4 a_{2}^{2}}} \\
& \cdot \tanh \left(\sqrt{\frac{(l b)^{2}-4 a_{2}\left(a_{1} \lambda+a_{5} l^{2}\right)}{8 a_{2}} z}\right),
\end{aligned}
$$




$$
\begin{aligned}
v_{T 1}= & \frac{l b}{2 a_{2}} z-\left(\sqrt{\frac{3}{2 a_{2}} \ln -\text { sech }}\right. \\
& \left.\left(\frac{1}{2} \sqrt{\frac{l^{2} b^{2}-4 a_{2}\left(a_{5} l^{2}+a_{1} \lambda\right)}{2 a_{2}} z}\right)^{2}\right)+d_{15}, \\
w_{T 2}= & \frac{l b}{2 a_{2}}-\sqrt{\frac{3(l b)^{2}-12 a_{2}\left(a_{1} \lambda+a_{5} l^{2}\right)}{4 a_{2}^{2}}} \\
& \cdot \tanh \left(-\sqrt{\frac{(l b)^{2}-4 a_{2}\left(a_{1} \lambda+a_{5} l^{2}\right)}{8 a_{2}}} z\right), \\
v_{T 2}= & \left.\left.\frac{l b}{2 a_{2}} z+\sqrt{\frac{3}{2 a_{2}}} z\right)^{2}\right) \\
& \cdot \ln \left(-\operatorname{sech}\left(\frac{1}{2} \sqrt{\frac{l^{2} b^{2}-4 a_{2}\left(a_{5} l^{2}+a_{1} \lambda\right)}{2 a_{2}}} z\right.\right. \\
& +d_{16},
\end{aligned}
$$

where $d_{15}$ and $d_{16}$ are integral constants.

5.3. Proof of Theorem 3. The sine-cosine method applies to the following form:

$$
w(z)=\left\{\lambda_{1} \cos ^{\beta_{1}}(\mu z)\right\}, \quad|z|<\frac{\pi}{2 \mu},
$$

or another form

$$
w(z)=\left\{\lambda_{1} \sin ^{\beta_{1}}(\mu z)\right\}, \quad|z|<\frac{\pi}{\mu},
$$

otherwise, it is zero; the values of $\lambda_{1}, \mu$, and $\beta_{1}$ parameters were measured.

We substitute

$$
w(z)=\left\{\lambda_{1} \cos ^{\beta_{1}}(\mu z)\right\}
$$

into equation (4) and obtain

$$
\begin{aligned}
\lambda_{1} & \beta_{1} \mu^{2}\left(\beta_{1}-1\right) \cos ^{\beta_{1}-2}(\mu z) \\
& -\left[\lambda_{1} \beta_{1}^{2} \mu^{2}-\left(a_{1} \lambda+a_{5} l^{2}\right) \lambda_{1}\right] \cos ^{\beta_{1}}(\mu z) \\
& +\frac{l b}{2} \lambda_{1}^{2} \cos ^{2 \beta_{1}}(\mu z)-\frac{a_{2}}{3} \lambda_{1}^{3} \cos ^{3 \beta_{1}}(\mu z)-\delta=0 .
\end{aligned}
$$

For the cosine functions of the same homomorphic power, extract its undetermined coefficients and set them to zero, and the following equations can be obtained:

$$
\left\{\begin{array}{l}
\beta_{1}-1 \neq 0, \\
\beta_{1}-2=3 \beta_{1}, \\
\lambda_{1} \beta_{1} \mu^{2}\left(\beta_{1}-1\right)=\frac{a_{2}}{3} \lambda_{1}^{3}, \\
\left(a_{1} \lambda+a_{5} l^{2}\right) \lambda_{1}=-\lambda_{1} \beta_{1}^{2} \mu^{2}, \\
\frac{l b}{2} \lambda_{1}^{2}=0 .
\end{array}\right.
$$

Solving the above simultaneous equations can get

$$
\begin{aligned}
& \beta_{1}=-1, \\
& \mu= \pm \sqrt{-a_{1} \lambda-a_{5} l^{2}}, \\
& \lambda_{1}= \pm \sqrt{\frac{-6\left(a_{1} \lambda+a_{5} l^{2}\right)}{a_{2}} .}
\end{aligned}
$$

In the end, we get the result

$$
\begin{aligned}
w_{c 1}(z)= & \sqrt{\frac{-6 a_{5} l^{2}-6 a_{1} \lambda}{a_{2}}} \sec \left(\sqrt{-a_{5} l^{2}-a_{1} \lambda} z\right), \\
v_{c 1}(z)= & \int w_{c 1}(z) d z=\sqrt{\frac{6}{a_{2}}} \ln \left(\sec \left(\sqrt{-a_{5} l^{2}-a_{1} \lambda} z\right)\right. \\
& \left.+\tan \left(\sqrt{-a_{5} l^{2}-a_{1} \lambda} z\right)\right)+d_{17},
\end{aligned}
$$

$$
\begin{aligned}
w_{c 2}(z)= & -\sqrt{\frac{-6 a_{5} l^{2}-6 a_{1} \lambda}{a_{2}}} \sec \left(\sqrt{-a_{5} l^{2}-a_{1} \lambda z}\right), \\
v_{c 2}(z)= & \int w_{c 2}(z) d z=\sqrt{\frac{6}{a_{2}}} \ln \left(\sec \left(\sqrt{-a_{5} l^{2}-a_{1} \lambda} z\right)\right. \\
& \left.+\tan \left(\sqrt{-a_{5} l^{2}-a_{1} \lambda} z\right)\right)+d_{18},
\end{aligned}
$$

where $d_{17}$ and $d_{18}$ are integral constants.

We substitute

$$
w(z)=\left\{\lambda_{1} \sin ^{\beta_{1}}(\mu z)\right\}
$$

into equation (4) and obtain

$$
\begin{aligned}
& \lambda_{1} \beta_{1} \mu^{2}\left(\beta_{1}-1\right) \sin ^{\beta_{1}-2}(\mu z)-\left[\lambda_{1} \beta_{1}^{2} \mu^{2}-\left(a_{1} \lambda+a_{5} l^{2}\right) \lambda_{1}\right] \\
& \quad \cdot \sin ^{\beta_{1}}(\mu z)+\frac{l b}{2} \lambda_{1}^{2} \sin ^{2 \beta_{1}}(\mu z)-\frac{a_{2}}{3} \lambda_{1}^{3} \sin ^{3 \beta_{1}}(\mu z)-\delta=0 .
\end{aligned}
$$




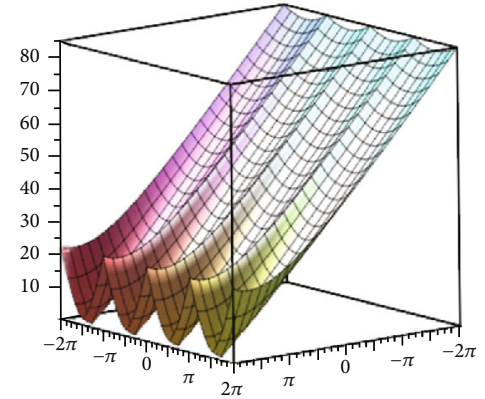

(a)

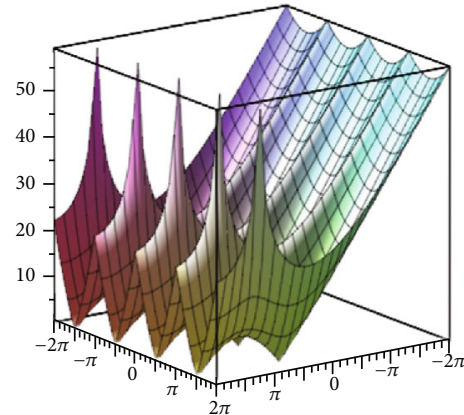

(b)

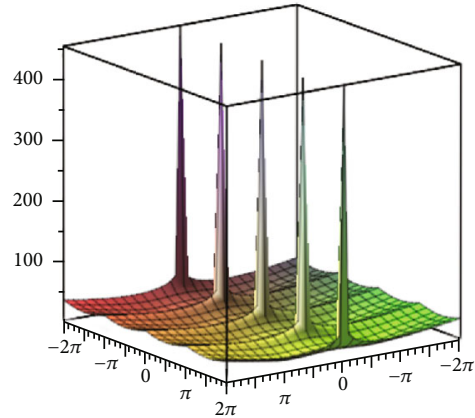

(c)

FIGURE 1: The three-dimensional images of $v_{11}(z)$ in three situations.

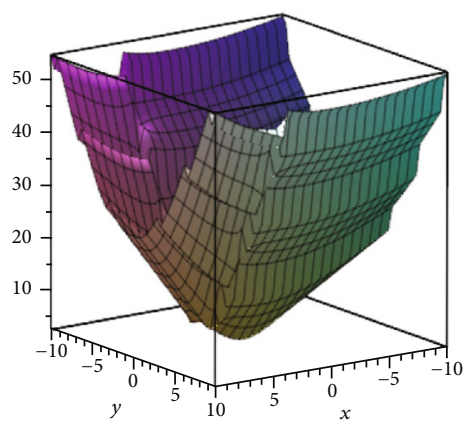

(a)

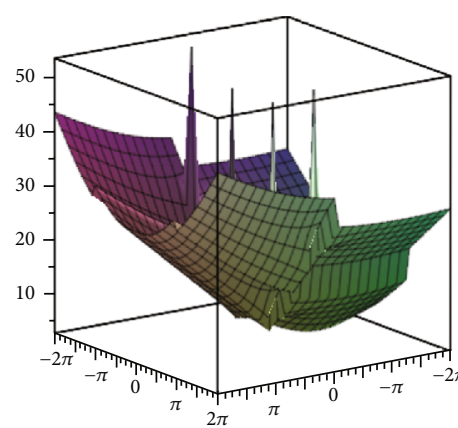

(b)

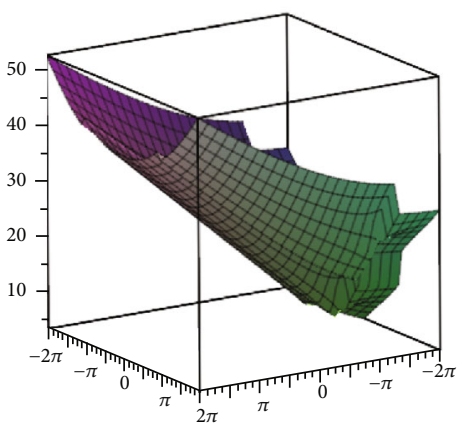

(c)

FIgURE 2: The three-dimensional images of $v_{T 1}(z)$ in three situations.

For the sine functions of the same homomorphic power, extract its undetermined coefficients and set them to zero, and the following equations can be obtained:

$$
\left\{\begin{array}{l}
\beta_{1}-1 \neq 0, \\
\beta_{1}-2=3 \beta_{1}, \\
\lambda_{1} \beta_{1} \mu^{2}\left(\beta_{1}-1\right)=\frac{a_{2}}{3} \lambda_{1}^{3}, \\
\left(a_{1} \lambda+a_{5} l^{2}\right) \lambda_{1}=-\lambda_{1} \beta_{1}^{2} \mu^{2}, \\
\frac{l b}{2} \lambda_{1}^{2}=0 .
\end{array}\right.
$$

Solving the above simultaneous equations can get

$$
\begin{aligned}
& \beta_{1}=-1, \\
& \mu= \pm \sqrt{-a_{1} \lambda-a_{5} l^{2},} \\
& \lambda_{1}= \pm \sqrt{\frac{-6\left(a_{1} \lambda+a_{5} l^{2}\right)}{a_{2}} .}
\end{aligned}
$$

In the end, we get the result:

$$
w_{s 1}(z)=\sqrt{\frac{-6 a_{5} l^{2}-6 a_{1} \lambda}{a_{2}}} \csc \left(\sqrt{-a_{5} l^{2}-a_{1} \lambda} z\right),
$$

$$
\begin{aligned}
v_{s 1}(z)= & \int w_{s 1}(z) d z=-\sqrt{\frac{6}{a_{2}}} \ln \left(\csc \left(\sqrt{-a_{5} l^{2}-a_{1} \lambda} z\right)\right. \\
& \left.+\cot \left(\sqrt{-a_{5} l^{2}-a_{1} \lambda} z\right)\right)+d_{19}, \\
w_{s 2}(z)= & -\sqrt{\frac{-6 a_{5} l^{2}-6 a_{1} \lambda}{a_{2}}} \csc \left(\sqrt{-a_{5} l^{2}-a_{1} \lambda} z\right), \\
v_{s 2}(z)= & \int w_{s 2}(z) d z=\sqrt{\frac{6}{a_{2}}} \ln \left(\csc \left(\sqrt{-a_{5} l^{2}-a_{1} \lambda} z\right)\right. \\
& \left.+\cot \left(\sqrt{-a_{5} l^{2}-a_{1} \lambda} z\right)\right)+d_{20},
\end{aligned}
$$

where $d_{19}$ and $d_{20}$ are integral constants.

\section{Computer Simulations}

In this section, we use computer simulation images to explain the results obtained according to three different methods and further analyze the nature of the simple periodic solutions $v_{11}(z), \quad v_{T 1}(z)$, and $v_{c 1}(z)$ in the $(2+1)$-dimensional Jaulent-Miodek equation.

Figure 1 shows the three-dimensional images of $v_{11}(z)$ by considering the values $a_{1}=2, a_{2}=2, a_{5}=2, l=2, b=5, \lambda=2$, 


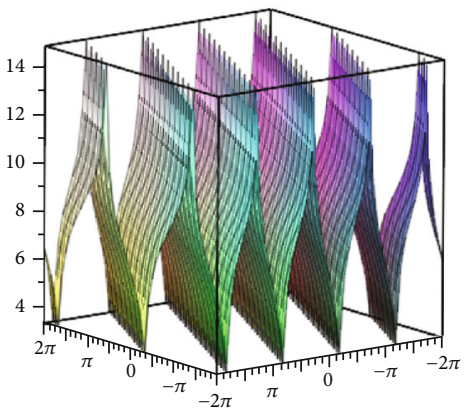

(a)

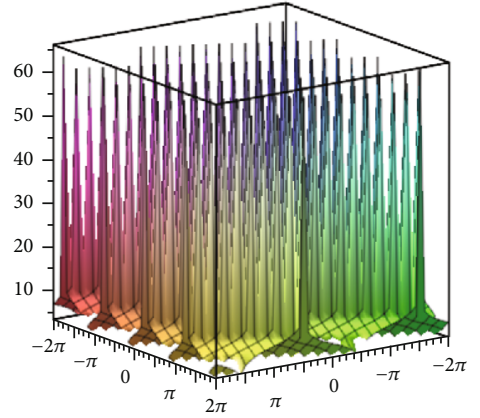

(b)

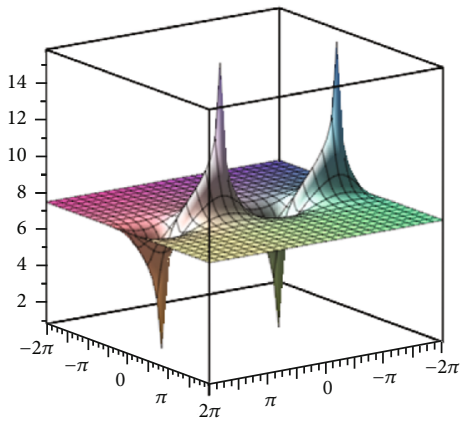

(c)

FIgURE 3: The three-dimensional images of $v_{c 1}(z)$ in three situations.

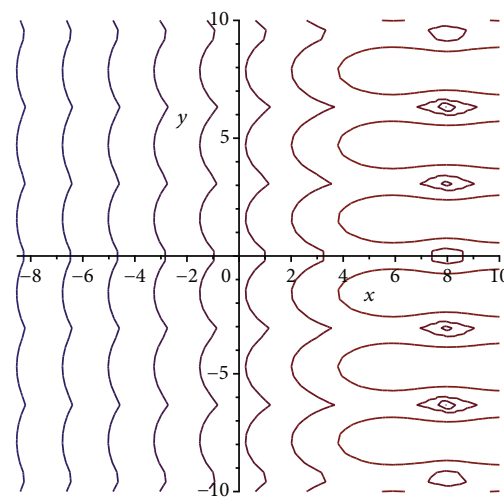

(a)

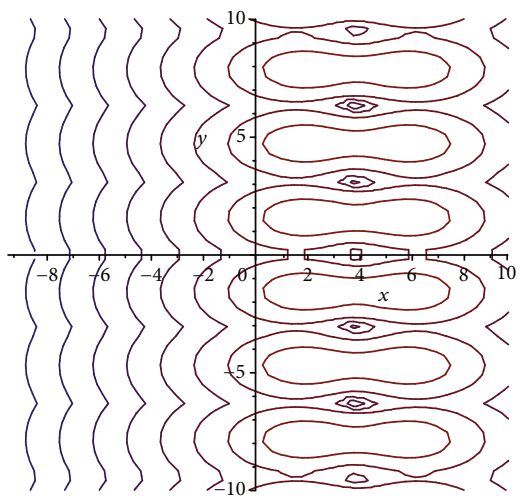

(b)

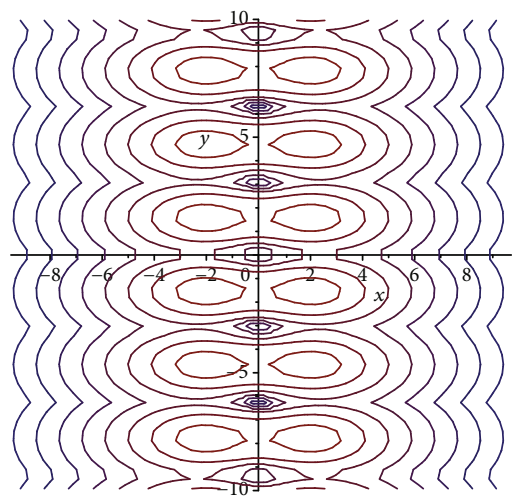

(c)

FIGURE 4: The two-dimensional images of $v_{11}(z)$ in three situations.

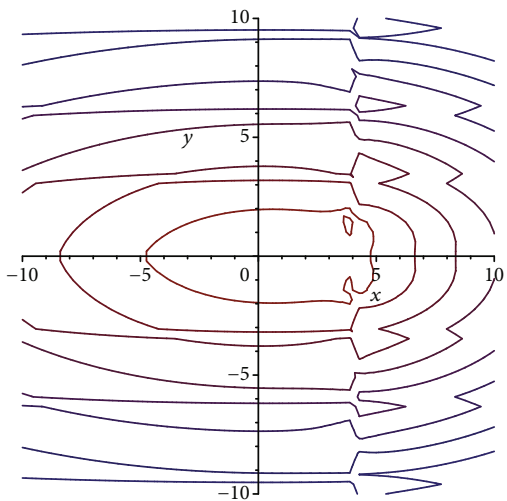

(a)

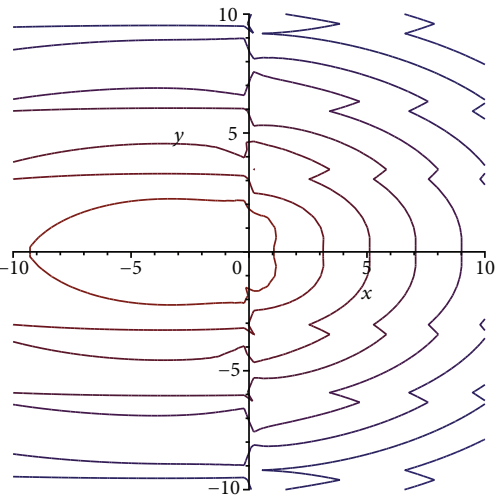

(b)

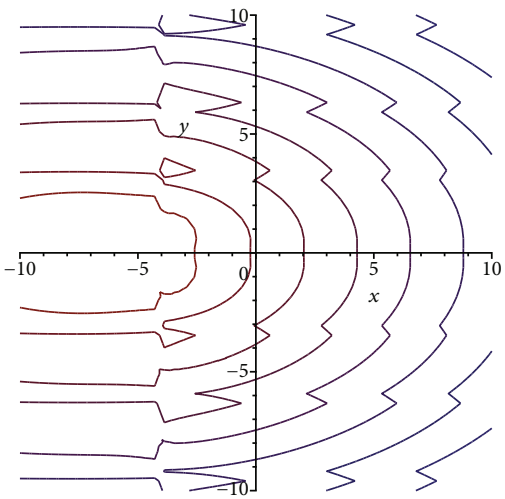

(c)

Figure 5: The two-dimensional images of $v_{T 1}(z)$ in three situations.

$c=2, \delta_{1}=3, \mu=2$, and $d_{1}=7$; from Figures $1(\mathrm{a})-1(\mathrm{c}), t$ takes the following three different values: $t=-2, t=0$, and $t=2$. Figures $1(\mathrm{a})-1(\mathrm{c})$ demonstrate discontinuities of solution $v_{11}(z)$ on the domain.

Figure 2 shows the three-dimensional images of $v_{T 1}(z)$ by considering the values $a_{1}=2, a_{2}=2, a_{5}=2, l=2, b=5, \lambda=2$, and $d_{15}=7$; from Figures $2(\mathrm{a})-2(\mathrm{c}), t$ takes the following three different values: $t=-2, t=0$, and $t=2$. Figures $2(\mathrm{a})-$ 2(c) describe the kink wave and parabola solution to the nonlinear evolution equation (3).

Figure 3 shows the three-dimensional images of $v_{c 1}(z)$ by considering the values $a_{1}=3, a_{2}=2, a_{5}=2, l=2, b=5$, $\lambda=-3$, and $d_{19}=7$; from Figures $3(\mathrm{a})-3(\mathrm{c}), t$ takes the following three different values: $t=-2, t=0$, and $t=2$. Figures $3(\mathrm{a})-3(\mathrm{c})$ show the singularities on the domain. However, when $t>0$, the image changes dramatically. 


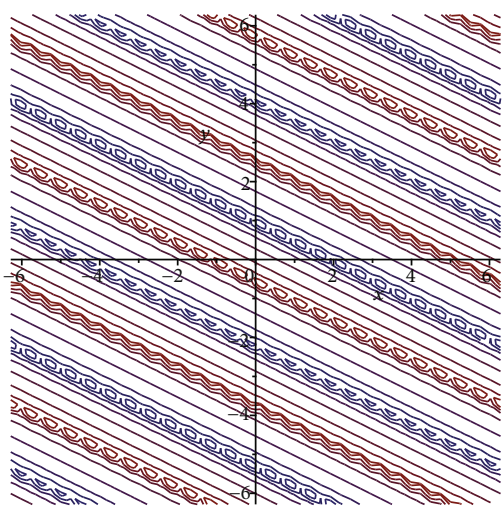

(a)

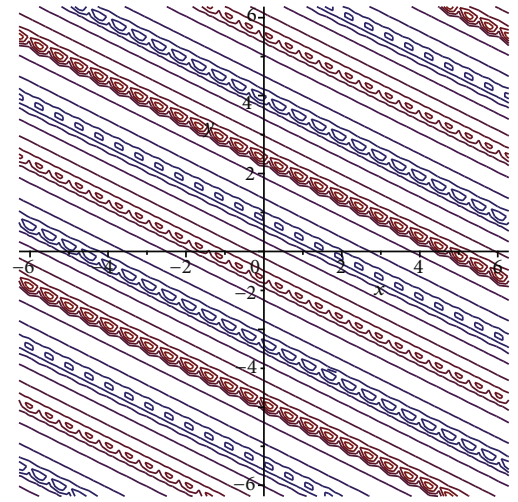

(b)

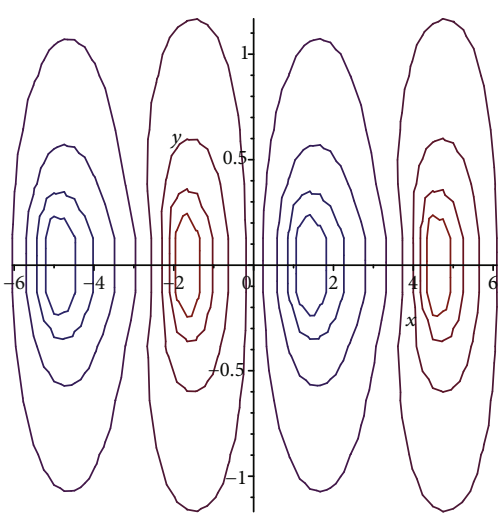

(c)

Figure 6: The two-dimensional images of $v_{c 1}(z)$ in three situations.

Figure 4 shows the two-dimensional images of $v_{11}(z)$ by considering the values $a_{1}=2, a_{2}=2, a_{5}=2, l=2, b=5, \lambda=$ 2, $c=2, \delta_{1}=3, \mu=2$, and $d_{1}=7$; from Figures 4(a) $-4(\mathrm{c})$, $t$ takes the following three different values: $t=-2, t=0$, and $t=2$.

Figure 5 shows the two-dimensional images of $v_{T 1}(z)$ by considering the values $a_{1}=2, a_{2}=2, a_{5}=2, l=2, b=5, \lambda=2$, and $d_{15}=7$; from Figures 5(a)-5(c), $t$ takes the following three different values: $t=-2, t=0$, and $t=2$.

Figure 6 shows the two-dimensional images of $v_{c 1}(z)$ by considering the values $a_{1}=3, a_{2}=2, a_{5}=2, l=2, b=5, \lambda=$ -3 , and $d_{19}=7$; from Figures $6(\mathrm{a})-6(\mathrm{c}), t$ takes the following three different values: $t=-2, t=0$, and $t=2$.

The above two-dimensional images all intuitively and accurately reflect the periodicity of function fluctuations.

\section{Discussions and Conclusions}

By the $\exp (-\phi(z))$-expansion method, we have obtained fourteen forms of solutions to the Jaulent-Miodek equation. Utilizing the tanh method, we obtained four forms of solutions of the Jaulent-Miodek equation. In the end, utilizing the sine-cosine method, we obtained two forms of solutions to the Jaulent-Miodek equation. Combining the existing results found in this paper, more results are easily obtained by the $\exp (-\phi(z))$-expansion method.

In [35], the authors adopt this complex method to seek for analytic solution of equation (3); the elliptic function solution is obtained by the complex method. From our paper and [35], it is easy to see that by utilizing the $\exp (-\phi(z))$ expansion method and complex method, both can obtain the hyperbolic function solutions for the Jaulent-Miodek equation. Unfortunately, utilizing the sine-cosine approach, we find only the trigonometric function solutions for the Jaulent-Miodek equation.

In [37], the authors used both methods, namely, the $G^{\prime}$ / $G^{2}$-expansion method and the Jacobi elliptic equation method to calculate and analyze the explicit exact traveling wave solutions of the $(2+1)$-dimensional Jaulent-Miodek equation as given by equation (1). By applying the $G^{\prime} / G^{2}$ expansion method to the equation, the solutions were obtained by the author including the triangular hyperbolic function solutions and rational function solutions. Implemented by the Jacobi elliptic equation method (analytical equation (1)), three sets of parameter values were reached. Every set has produced fifteen exact terms for the solution of Jacobi elliptic function solutions. By comparing the methods to [37], the methods in our article seem more concise and straightforward.

All three methods in this paper have their own characteristics. We trust that the above method presented here and the methods in $[35,37]$ together can be applied more effectively to find out solutions for nonlinear evolution equations currently and henceforth. The above-mentioned methods are very helpful tools for unearthing exact solutions to the nonlinear evolution equations.

\section{Data Availability}

No data were used to support this study.

\section{Conflicts of Interest}

The authors declare that they have no conflicts of interest.

\section{Authors' Contributions}

All authors typed, read, and approved the final manuscript.

\section{Acknowledgments}

The work presented in this paper is supported by the Plateau Disciplines in Shanghai. Also, this work was supported by the Leading Academic Applied Mathematical of Shanghai Dianji University (16JCXK02) and Humanity and Social Science Youth Foundation of Ministry of Education (18YJC630120). The work also presented in this paper is supported by the Shanghai Multi-Direction Dieforging Engineering Technology Research Center (No. 20DZ2253200) and supported by the Science and Technology Support Project in the Biomedical Field of "Science and Technology Innovation Action Plan" of Shanghai in 2019 (No. 19441914900). 


\section{References}

[1] D. K. Campbell and Y. N. Huang, "Nonlinear science from sample to practical," Mechanical Progress, vol. 19, no. 4, pp. 90-100, 1989.

[2] J. L. Pinto de Sa and M. Louro, "On human life risk-assessment and sensitive ground fault protection in MV distribution networks," IEEE Transactions on Power Delivery, vol. 25, no. 4, pp. 2319-2327, 2010.

[3] C. S. Liu and L. C. Jiao, "Application of nonlinear programming in reactive power compensation optimization of mine high-voltage distribution network," Journal of Coal, vol. 27, no. 4, pp. 444-448, 2002.

[4] C. Z. Tian, L. L. Wang, and Y. Yang, "Distribution network evaluation method combined with nonlinear mapping and clustering analysis," Journal of Power Systems and Automation, vol. 30, no. 12, pp. 145-150, 2018.

[5] D. W. Lai and Z. X. Wang, "Some nonlinear micropartial equations appearing in power system," Applied Mathematics, vol. 3, no. 1, pp. 84-90, 1990.

[6] G. B. Xiang, "Asymmetric nonlinear oscillation in parallel operation of power stations," ACTA Automatica Sinica, vol. 15, no. 6, pp. 552-556, 1989.

[7] C. de Marco and A. R. Bergen, "A security measure for random load disturbances in nonlinear power system models," IEEE Trans on Circuits and Systems, vol. 34, no. 12, pp. 15461557, 1987.

[8] W. B. Xu and J. Wang, "Stability analysis of random small disturbances in nonlinear power systems," Power System Technology, vol. 38, no. 10, pp. 2735-2740, 2014.

[9] X. H. Lu and Y. T. Zheng, "Power system load forecasting model based on nonlinear time series snalysis," Power and Energy, vol. 37, no. 2, pp. 197-201, 2015.

[10] P. X. Song, Y. H. Li, and P. Wang, "Research on local approximation method of transient stability region boundary of power system," Journal of Electrical Engineering and Control, vol. 20, no. 5, pp. 7-13, 2016.

[11] C. S. Gardner, J. M. Greene, M. D. Kruskal, and R. M. Miura, "Method for solving the Korteweg-deVries equation," Physical Review Letters, vol. 19, no. 19, pp. 1095-1097, 1967.

[12] M. L. Wang and Z. B. Li, "The principle of homogeneous balance and its application," Journal of LanZhou University, vol. 35, no. 3, pp. 8-16, 1999.

[13] J. P. Yu and Y. L. Sun, "Lump solutions to dimensionally reduced Kadomtsev-Petviashvili-like equations," Nonlinear Dynamics, vol. 87, no. 2, article 3122, pp. 1405-1412, 2017.

[14] J. P. Yu and Y. L. Sun, "A direct Bäcklund transformation for a $(3+1)$-dimensional Kadomtsev-Petviashvili-Boussinesq-like equation," Nonlinear Dynamics, vol. 90, no. 4, pp. 22632268, 2017.

[15] M. Matinfar and K. Hosseini, "Optical solitons of $(2+1)$ dimensional nonlinear Schrodinger equation involving linear and nonlinear effects," Optik-International Journal for Light and Electron Optics, vol. 228, no. 23, article 166110, 2021.

[16] L. Akinyemi, K. Hosseini, and S. Salahshour, "The bright and singular solitons of $(2+1)$-dimensional nonlinear Schrodinger equation with spatio-temporal dispersions," Optik-International Journal for Light and Electron Optics, vol. 242, no. 7, article 167120, 2021.

[17] M. L. Wang, Y. M. Wang, and Y. B. Zhou, "An auto-Backlund transformation and exact solutions to a generalized KdV equa- tion with variable coefficients and their applications," Physics Letters A, vol. 303, no. 1, pp. 45-51, 2002.

[18] W. Hereman and M. Takaoka, "Solitary wave solutions of nonlinear evolution and wave equations using a direct method and macsyma," Journal of Physics A: Mathematical and General, vol. 23, no. 21, pp. 4805-4822, 1990.

[19] J. Weiss, M. Tabor, and G. Carnevale, "The Painlevé property for partial differential equations," Journal of Mathematical Physics, vol. 24, no. 3, pp. 522-526, 1983.

[20] D. G. Zhang, "Integrability of fermionic extensions of the Burgers equation," Physics Letters A, vol. 223, no. 6, pp. 436438, 1996.

[21] C. S. Liu, "Trial equation method to nonlinear evolution equations with rank inhomogeneous: mathematical discussions and its applications," Communications in Theoretical Physics, vol. 45, no. 2, pp. 219-223, 2006.

[22] B. Ren, W. X. Ma, and J. Yu, "Characteristics and interactions of solitary and lump waves of a $(2+1)$-dimensional coupled nonlinear partial differential equation," Nonlinear Dynamics, vol. 96, no. 1, pp. 717-727, 2019.

[23] B. Ren, J. Lin, and Z. M. Lou, "Consistent Riccati expansion and rational solutions of the Drinfel'd-Sokolov-Wilson equation," Applied Mathematics Letters, vol. 105, article 106326, no. $105,2020$.

[24] J. Satsuma, "Hirota bilinear method for nonlinear evolution equations," Direct and Inverse Methods in Nonlinear Evolution Equations, vol. 632, pp. 171-222, 2003.

[25] J. M. Zuo and Y. M. Zhang, "The Hirota bilinear method for the coupled Burgers equation and the high-order BoussinesqBurgers equation," Chinese Physics B, vol. 20, no. 1, pp. 010205-010279, 2011.

[26] M. Jaulent and I. Miodek, "Nonlinear evolution equations associated with 'enegry-dependent Schrodinger potentials'," Letters in Mathematical Physics, vol. 1, no. 3, pp. 243-250, 1976.

[27] D. H. Feng and J. B. Li, "Traveling wave solution branch of Jaulent-Miodek equation," Applied Mathematics and Mechanics, vol. 28, no. 8, pp. 894-900, 2007.

[28] A. M. Wazwaz, "Multiple kink solutions and multiple singular kink solutions for $(2+1)$-dimensional nonlinear models generated by the Jaulent-Miodek hierarchy," Physics Letters A, vol. 373, no. 21, pp. 1844-1846, 2009.

[29] H. C. Cai, Z. Y. Qin, and A. P. Deng, "Symmetry transformation and new exact multiple kink and singular kink solutions for $(2+1)$-dimensional nonlinear models generated by the Jaulent-Miodek hierarchy," Communications in Theoretical Physics, vol. 59, no. 2, pp. 141-145, 2013.

[30] H. C. Ma, A. P. Deng, and Y. D. Yu, "Lie symmetry group of $(2+1)$-dimensional Jaulent-Miodek equation," Thermal Science, vol. 18, no. 5, pp. 1547-1552, 2014.

[31] M. Matinfar, M. Eslami, and S. Roshandel, "The first integral method to study the $(2+1)$-dimensional Jaulent-Miodek equations," Pramana, vol. 85, no. 4, pp. 593-603, 2015.

[32] Y. Li, X. Q. Liu, and X. P. Xin, "Explicit solutions and conservation laws of the extended $(2+1)$-dimensional JaulentMiodek equation," Journal of Hebei Normal University, vol. 40, no. 5, pp. 376-384, 2016.

[33] F. Q. Wei, S. H. Zhu, and X. J. Wang, "Kink soliton solutions of the $(2+1)$-dimensional Jaulent-Miodek equation," Journal of China West Normal University, vol. 38, no. 42, pp. 392-397, 2017. 
[34] T. Motsepa, M. Abudiab, and C. Masood Khalique, "A study of an extended generalized $(2+1)$-dimensional Jaulent-Miodek equation," International Journal of Nonlinear Sciences and Numerical Simulation, vol. 19, no. 3-4, pp. 391-395, 2018.

[35] Y. Y. Gu, B. M. Deng, and J. M. Lin, "Exact traveling wave solutions to the $(2+1)$-dimensional Jaulent-Miodek equation," Advances in Mathematical Physics, vol. 2018, no. 43, Article ID 5971646, 9 pages, 2018.

[36] M. S. M. Shehata and E. H. M. Zahran, "The solitary wave solutions of important model in particle physics and engineering according to two different techniques," American Journal of Computational Mathematics, vol. 9, no. 9, pp. 317-327, 2019.

[37] S. Kaewta, S. Sirisubtawee, and N. Khansai, "Explicit exact solutions of the $(2+1)$-dimensional integro-differential Jaulent-Miodek evolution equation using the reliable methods," International Journal of Mathematics and Mathematical Sciences, vol. 2020, no. 2, Article ID 2916395, 19 pages, 2020.

[38] X. Geng, C. Cao, and H. H. Dai, "Quasi-periodic solutions for some $(2+1)$-dimensional integrable models generated by the Jaulent-Miodek hierarchy," Mathematical and General, vol. 34, no. 5, pp. 989-1004, 2001.

[39] Y. Y. Zhang, X. Q. Liu, and G. W. Wang, "Symmetry reductions and exact solutions of the $(2+1)$-dimensional JaulentMiodek equation," Applied Mathematic and Computation, vol. 219, no. 3, pp. 911-916, 2012.

[40] W. Malfliet and W. Hereman, "The tanh method: II. Perturbation technique for conservative systems," Physica Scripta, vol. 54, no. 6, pp. 569-575, 1996.

[41] A. M. Wazwaz, "Compact and noncompact physical structures for the ZK-BBM equation," Applied Mathematics and Computation, vol. 169, no. 1, pp. 713-725, 2005.

[42] M. T. Alquran, "Solitons and periodic solutions to nonlinear partial differential equations by the sine-cosine method," Applied Mathematics and Information Sciences, vol. 6, no. 1, pp. 85-88, 2012. 